\title{
Ezequiel A. Chávez y la secularización del adolescente mexicano: Ensayo de psicología de la adolescencia (1928)
}

\author{
Ezequiel A. Chávez and the Secularization of the Mexican Adolescent: \\ Ensayo de psicología de la adolescencia (1928) \\ Ivonne Meza Huacuja \\ INSTITUTO DE INVESTIGACIONES SOBRE LA UNIVERSIDAD Y LA EDUCACIÓN-UNAM, \\ ivy.meza@gmail.com
}

A finales del siglo xIx fueron introducidas en México, a partir del sistema educativo, nuevas definiciones sobre la adolescencia basadas en observaciones científicas, concebidas desde la perspectiva de la naciente psicología experimental y de la medicina. El presente artículo está enfocado en el estudio de las propuestas del profesor, funcionario y "psicólogo" mexicano Ezequiel A. Chávez sobre la formación de los adolescentes. Mi objetivo principal se centra en el análisis y recepción de su libro intitulado Ensayo de psicología de la adolescencia (1928), donde su autor compiló sus cursos de "psicología de la adolescencia" impartidos en la Escuela Normal Superior desde 1922. El texto que a continuación presento es un estudio de caso donde se advierte que las conceptualizaciones de la adolescencia deben concebirse históricamente, ya que reflejan las preocupaciones, credos, proyectos sociales y nacionales de los grupos que han contribuido a generar sus distintos significados.

PaLABRAS Clave: adolescencia, educación, Porfirismo, Revolución mexicana, psicología.

In the late $19^{\text {th }}$ century, new definitions of adolescence were introduced in Mexico through the educational system, novel descriptions based on scientific observations conceived from the perspective of the nascent fields of experimental psychology and medicine. The present study analyzes the proposals for adolescent education put forth by the Mexican professor, politician and psychologist Ezequiel A. Chávez. The main goal is to elucidate the impact of his book Ensayo de psicologia de la adolescencia (An Assay in the Psychology of Adolescence, 1928), which was based on his courses on the "psychology of adolescence" given at the Escuela Normal Superior beginning in 1922. The article presents a case study which suggests that concepts of adolescence must be conceived historically because they reflect the concerns, beliefs and social and national projects of the groups involved in generating their different meanings.

KeYwORDs: adolescence, education, Porfirian Mexico, Mexican Revolution, psychology.

Fecha de recepción del artículo: 16 de junio de 2015 / Fecha de aprobación: 6 de agosto de 2015 / Fecha de recepción de la versión final: 12 de agosto de 2015 


\section{A} unque el término espańol "adolescencia" aparece en algunas obras literarias y en tratados médicos de la antigüedad, las fuentes señalan que desde finales del siglo xIx fue utilizado para referirse a una etapa de la vida distinguida de la infancia y de la edad adulta por cambios fisiológicos, como el desarrollo de caracteres sexuales y psicológicos; como la rebeldía y la ensońación; la tendencia a congregarse en grupos; a enamorarse fácilmente, entre otras particularidades. La maleabilidad y susceptibilidad de la adolescencia, sobre la que psicólogos y pedagogos europeos y estadounidenses advirtieron, resultó ser un factor de alarma y una preocupación que alertó a ciertos sectores sociales. Los adolescentes de ambos sexos se convirtieron en sujetos estratégicos para ciertos individuos e instituciones en México. Su formación y educación fue disputada por el poder estatal y asociaciones religiosas. Aunque no fue el único factor que incidió en el impulso de los estudios sobre la niñez y la adolescencia, el advenimiento de la psicología experimental durante el siglo XIX contribuyó a la elaboración de políticas públicas, prioritariamente educativas, enfocadas en este último grupo etario en los Estados Unidos, Alemania, Inglaterra, Francia y México. ${ }^{1}$

Mi objetivo con el presente artículo es rescatar las aportaciones de Ezequiel A. Chávez (1868-1946), considerado por algunos especialistas como uno de los pioneros en la introducción de la psicología experimental en México, en la construcción del moderno significado de adolescencia en nuestro país. ${ }^{2} \mathrm{Mi}$ punto de partida será la exposición de las labores realizadas por Chávez al interior de las instituciones gubernamentales y educativas para así entender los medios de los que se sirvió para difundir los estudios sobre la adolescencia e impul-

${ }^{1}$ Principios de psicología fisiológica (1874) escrito por Wilhelm Wundt es considerado por algunos autores como el primer libro de psicología experimental. En este texto Wundt se proponía establecer a la psicología como una ciencia nueva. Para dar legitimidad a esta novel disciplina su autor agregaba que el punto de partida de sus observaciones provenían de fundamentos anatómicos y fisiológicos. Ludy T. Benjamin Jr., A Brief Study of Modern Psychology (Malden: Blackwell Publishing, 2007), 37.

${ }^{2}$ Rubén Ardila, La psicología en América latina: pasado presente y futuro (México: Siglo XXI editores, 1998), 34. Lucy María Reidl Martínez y María de Lourdes Echeveste García, La Facultad de Psicología de la Universidad Nacional Autónoma de México: treinta años a la vanguardia (México: UNAM, 2003), 76. 
sar lo que en ese momento se consideraban como medidas apropiadas para el tratamiento de los adolescentes de nuestro país. Me centraré en el estudio de su libro Ensayo de psicología de la adolescencia publicado en 1928, considerado como el primer texto elaborado en México que recopiló los estudios y preocupaciones que internacionalmente se debatían sobre esa edad. La importancia de dicho documento es que, para algunos autores, además de constituir la primera obra escrita por un hispanoamericano en psicología experimental representa un intento por construir una tipología sobre la adolescencia y los adolescentes mexicanos. Para los historiadores de hoy es una fuente importante para entender las expectativas e inquietudes que se tenían sobre dicho grupo etario en un contexto particular.

\section{Ezequiel A. Chávez: una psicología DE LA RECONCILIACIÓN}

Ezequiel Adeodato Chávez Lavista nació el 19 de septiembre de 1868 en Aguascalientes. Fue hijo del doctor Ignacio T. Chávez, exgobernador de dicho estado, que con su familia se mudó a la Ciudad de México para ejercer como diputado por su demarcación en 1876. El joven Chávez realizó sus estudios en el Instituto AngloFranco-Mexicano, en la Escuela Nacional Preparatoria (ENP) y en la Escuela Nacional de Jurisprudencia. Fue alumno de Ignacio Manuel Altamirano, Justo Sierra, Rafael Barba, Manuel María Contreras y Miguel Schultz, por cuya recomendación ingresó como docente a esta última institución en $1891 .{ }^{3}$ Fue hasta 1893 cuando al sustituir al licenciado José María Gamboa en la cátedra de Lógica y Moral llamó su atención la psicología, tema que fue abordado en algunas sesiones de la materia impartida por su predecesor.

Desde 1895, obtuvo diversos puestos dentro de la Secretaría de Justicia e Instrucción Pública. Sin embargo, en 1905, cuando Justo Sierra ocupó el máximo cargo de dicha institución invitó a Chávez

${ }^{3}$ Schultz fue profesor de Geografía y recomendó a Chávez como su remplazo. Leticia Chávez, Recordando a mi padre. Ezequiel A. Chávez (México: Asociación Civil "Ezequiel A. Chávez", 1967), 17. 
como subdirector de la misma. En su nuevo puesto fue comisionado durante los ańos 1903, 1906 y 1909 para visitar algunas universidades en los Estados Unidos, con dicha información fundó la Universidad Nacional en 1910. ${ }^{4}$ En 1896, Chávez fue el responsable de la elaboración del plan de Estudios de la Escuela Nacional Preparatoria y, en 1903, propuso la introducción oficial de la Cátedra de Psicología en dicha institución. ${ }^{5}$

En 1907 se convirtió en el presidente de la efímera Sociedad Mexicana de Estudios Psicológicos, seis años después fue nombrado director de la Escuela de Altos Estudios. De 1913 a 1914 fue rector de la Universidad Nacional de México cargo que volvió a ocupar en 1923. Durante los ańos 1916 y 1917 vivió en los Estados Unidos en un autoexilio tras el derrocamiento de Victoriano Huerta.

La trayectoria de Chávez en la Escuela de Altos Estudios, que en 1910 pasó a formar parte de la Universidad, es importante para nuestros fines porque él fue quien diseńó aquel establecimiento cuyo objetivo inicial era el perfeccionamiento "de la instrucción obtenida en las escuelas profesionales, desarrollar investigación y preparar docentes para las propias dependencias universitarias" . ${ }^{6} \mathrm{Al}$ instaurarse en ella la cátedra de Psicología de la Adolescencia en 1921, cumplió con el segundo de los nuevos estatutos de la institución redactado por el mismo Chávez:

Por lo que atañe al perfeccionamiento de los profesores de escuelas secundarias y de las facultades -así como de los educadores de primaria- se instaurará un sistema de cursos que abarque un conjunto de materias que constituyan una formación con bases sólidas y permitan ampliar y precisar los conocimientos. ${ }^{7}$

${ }^{4}$ Javier Garciadiego Dantán, Rudos contra cientificos. La Universidad Nacional durante la Revolución Mexicana (México: El Colegio de México, 1996), 24-25.

${ }^{5}$ Charles Hale, La transformación del liberalismo en México a fines del siglo XIX (México: Fondo de Cultura Económica, 2002), 319.

${ }^{6}$ Javier Garciadiego Dantán "De Justo Sierra a Vasconcelos. La Universidad Nacional Durante la Revolución Mexicana”, Historia Mexicana, Homenaje a don Edmundo O’Gorman 46(4) (abril-junio 1997): 785.

${ }^{7}$ Claude Fell, José Vasconcelos: los años del águila, 1920-1925 (México: unAM, 2009), 295. 
En 1920 a petición del entonces rector de la Universidad Nacional de México, José Vasconcelos, elaboró una propuesta de restructuración de la educación nacional ante el consejo directivo de la Universidad al que llamó Proyecto de ley de federalización de la enseñanza mexicana y de creación de la Secretaría de Instrucción Pública Federal. Dicho documento, con enmendaduras realizadas por Vasconcelos, fue enviado por este último al Congreso, que lo aprobó al año siguiente. ${ }^{8}$

A pesar de sus planes de jubilación en 1924, Chávez siguió trabajando como profesor en México, Francia y Espańa. Justo en el ocaso de su vida intelectual escribió Ensayo de psicología de la adolescencia en 1928.

Las reflexiones vertidas por Chávez en Ensayo, además de acercarnos a algunas representaciones sobre la adolescencia durante un periodo en particular, nos ayudan a entender su sobrevivencia política y docente pese el cambio de régimen y durante los distintos gobiernos posrevolucionarios. Su permanencia en algunos cargos públicos respondió a la falta de experiencia de los funcionarios de las nuevas administraciones, lo que a su vez explica por qué el conflicto bélico no representó un punto de ruptura (total y abrupto) con el pasado porfirista. Es importante resaltar que la mencionada reconstrucción nacional posrevolucionaria fue un conglomerado de viejas y nuevas expectativas que se conjugaron en los nuevos discursos. ${ }^{9}$ De la misma forma, la posición filosófica ambigua de Chávez, entre el positivismo y el humanismo, contribuyó a que las reformas educativas, los cambios en los contenidos curriculares y la apertura de nuevas instituciones por él propuestas durante el periodo de transición entre un régimen y otro gozaran de vigencia y aceptación entre algunos funcionarios de los diferentes gobiernos. ${ }^{10}$

\footnotetext{
${ }^{8}$ Un estudio detallado sobre los contenidos del proyecto elaborado por Chávez puede encontrarse en Gustavo Meza Medina, La creación de la SEP: entre Chávezy Vasconcelos, ponencia presentada en el Ix Congreso Nacional de Investigación Educativa celebrado en Mérida Yucatán del 5 al 9 de noviembre de 2007.

${ }^{9}$ Alan Knight, "Popular Culture and the Revolutionary State in México, 19101940", Hispanic American Historical Review 74(3) (agosto 1994): 393-444.

${ }^{10}$ Recordemos que el positivismo fue asociado con el grupo porfirista de los "científicos”, y que el Ateneo de la Juventud, organización a la que perteneció José Vasconcelos,
} 
Chávez fue insistente en resaltar la importancia de la aplicación conjunta del conocimiento científico con elementos de una doctrina católica moralizadora en las políticas públicas educativas, pues, esperaba que ambos fueran empleados en la formación adecuada e integral de los adolescentes. ${ }^{11}$ Con estas acciones, Chávez también intentó contribuir con el interés generalizado en el plano nacional e internacional de restituir la paz por medio de la educación espiritual de los jóvenes en las naciones afectadas como México por los conflictos bélicos.

Ensayo no sólo representó un estudio profundo acerca de las últimas tendencias sobre la psicología de los adolescentes, pues, también incorporó algunas reflexiones sobre la necesidad de reorganizar el sistema educativo mexicano y constituyó una revisión conservadora de la historia nacional. Por otro lado, significó una puerta de entrada al país para las teorías pedagógicas y psicológicas internacionales en boga. De hecho, podemos afirmar que más que una psicología clínica o experimental Chávez promovió la aplicación de una psicología educativa no sólo para México sino también para el resto del mundo:

Este libro intenta traer su contribución al conocimiento de los adolescentes para que, unidos al cabo con sus educadores, se encaminen a la realización del fin último de todo esfuerzo educativo bien orientado y aporten, en consecuencia, su concurso, para organizar la armonía que urge imponer a las antagónicas fuerzas entre las cuales se divide el mundo; no sólo a

secretario de Educación Pública en 1921 fue un centro de resistencia frente a dicha corriente de pensamiento. Por otro lado, Chávez participó desde finales del siglo XIX y principios del veinte en la transformación del plan de estudios de la Escuela Nacional Preparatoria incorporando materias humanistas. Para más información véase Ivonne Meza Huacuja, "La edad difícil. Los adolescentes modernos en la ciudad de México (1876-1934)" (Tesis de doctorado, El Colegio de México, 2015), 102-126.

${ }^{11}$ Chávez explica en 1938 las razones que motivaron la apertura de la Escuela Nacional de Altos Estudios: "Cuando inicié ante la antigua Secretaría de Instrucción Pública y Bellas Artes hacia el año de 1908 la creación de un nuevo órgano de la educación nacional que se llamó primero Escuela Nacional de Altos Estudios y que ahora se llama [sic] Facultad de Filosofía y Letras mi objeto fundamental era conseguir que México tuviese un instituto encargado de formar a los profesores de las escuelas preparatorias y de las escuelas normales de la República. Leticia Chávez, Recordando a mi padre, 21. 
fin de evitar que éste llegue a desplazarse, como tantos ilustres pensadores lo temen, sino con el rehacer la cultura, restituyendo así la posibilidad de que todos los pueblos, y especialmente México progresen. ${ }^{12}$

Una característica del trabajo de Chávez fue su incesante mirada al exterior, así lo ejemplifican sus constantes congresos, viajes de estudio e impartición de cursos en universidades estadounidenses y europeas, como la de Stanford, la de Berkeley, la de Cincinnati, la de Oxford y la Central de Madrid; ${ }^{13}$ su intercambio epistolar con personajes de gran peso dentro de las disciplinas psicopedagógicas como Helen Bowyer, James Mark Baldwin y Ernest Carrol Moore -con quien mantuvo una estrecha amistad--; ${ }^{14}$ su asidua compra de novedosas publicaciones en librerías y editoriales de Estados Unidos y Francia -que donó a la Universidad Nacional y que tradujo al español-; la invitación para impartir cátedras en la Facultad de Altos Estudios de la Universidad entre 1910 y 1914 a investigadores extranjeros como Franz Boaz, James Mark Baldwin y Karl Friedrich Reiche. ${ }^{15}$ Todos estos esfuerzos respondieron al "internacionalismo

${ }^{12}$ Ezequiel A. Chávez, Ensayo de psicología de la adolescencia (México: Jus, 1956), 14.

${ }^{13}$ Las visitas de 1903, 1906 y 1909 a los Estados Unidos fueron planeadas para que Chávez realizara observaciones sobre la estructura y el funcionamiento de las universidades, en particular, la de Stanford y la de Berkeley, con miras a la reapertura de la Universidad Nacional de México. El autoexilio de 1913 lo llevó a tocar puertas en diferentes ciudades de la Unión Americana, finalmente, la Universidad de Cincinnati lo contrató como profesor de literatura latinoamericana. Antes de su regreso a México, fue comisionado por Alberto J. Pani para el estudio de los museos en Filadelfia, Nueva York y Washington. En 1927, realizó un viaje al vir Congreso Internacional de Psicología en Groninga, Holanda, y ofreció sus conferencias sobre la adolescencia en la Universidad Central de Madrid. Luis Anaya Merchant, Ezequiel A. Chávez. Una aproximación biográfica a la historiografia de la rectificación (Aguascalientes: Gobierno del Estado de Aguascalientes, Instituto Cultural de Aguascalientes, 2002), 102-103 y 128-133.

${ }^{14}$ Helen Bowyer fue una pedagoga estadounidense que realizó en 1922 la prueba Detroit de habilidad de aprendizaje a niños mexicanos por petición de Chávez. James Baldwin fue un psicólogo social estadounidense quien planteó que el medio social influía en la conducta del individuo. Ernest Carrol Moore fue doctor en Filosofía por la Universidad de Yale y director de la Southern Branch, de la Universidad de California en 1924.

${ }^{15}$ Franz Boaz, antropólogo germano-estadounidense, fue uno de los pioneros de la antropología moderna y ha sido considerado como el fundador de la disciplina en los Estados Unidos; James Mark Baldwin fue contratado para impartir un curso de psicosociología en la Escuela de Altos Estudios; Karl Friedrich Reiche fue un botánico chileno- 
del pensamiento humano" que él mismo definió en 1928 como "la integración y divulgación del pensamiento y las investigaciones realizadas a nivel internacional en pos del avance y mejoramiento de la humanidad". ${ }^{16}$ Chávez procuró incorporar a México dentro de dichos esfuerzos pues intentó que algunos de sus escritos -incluyendo Ensayo de psicología - fueran considerados una aportación mexicana a esta misión mundial.

La aparición del libro tuvo como inspiración la organización de una serie de conferencias sobre psicología de la adolescencia impartidas en 1927 en la Universidad Central en Madrid, España, que el mexicano ofreció en nueve sesiones y que tuvieron como objetivo: ${ }^{17}$

Ofrecer a los profesores de las escuelas secundaria, normales y superiores, y a quienes se estén preparando para llegar a serlo, un resumen de estudios y observaciones referentes a la psicología de los adolescentes a fin de contribuir a satisfacer la necesidad, cada vez más vivamente sentida, de encaminar a las nuevas generaciones para que, adquiriendo una verdadera cultura, colaboren de manera armónica en el progreso del mundo. ${ }^{18}$

\section{ENTRE DOS MUNDOS: EL ADOLESCENTE SEGÚN Ezequiel A. Chávez}

Antes de proseguir con la descripción de los contenidos de Ensayo, me parece pertinente ubicar a la obra de Chávez como un compendio ecléctico de obras de filosofía, psicología y fisiología de autores

alemán. Los trabajos historiográficos que hablan sobre la estancia de Boaz y Baldwin en México son bastante abundantes. Sin embargo, el artículo de Mauricio Tenorio Trillo es uno de los que mejor contextualizan y resumen sus trabajos en la Universidad Nacional. Mauricio Tenorio Trillo, "Stereophonic Scientific Modernisms: Social Science between Mexico and the United States, 1880s-1930s", The Journal of American History, The Nation Beyond: Transnational Perspectives on United States History: Special Issues 86(3) (diciembre 1999); 1,156-1,187.

${ }^{16}$ Ezequiel A. Chávez, Ensayo, 16.

${ }^{17}$ Archivo Histórico de la UnAm (AHUnam), Ezequiel A. Chávez, caja 125, exp. 249, doc. 2, foja 2, año 1927.

${ }^{18} \mathrm{La}$ intención de Chávez, a través de sus publicaciones fue llegar a lectores fuera de las fronteras nacionales. AHUNAM, Ezequiel A. Chávez, caja 125, exp. 249, doc. 2, foja 2, año 1927. 
extranjeros. La mayor parte de los textos señalados a lo largo del libro corresponden a primeras ediciones y lo último en investigaciones en el ramo psicopedagógico en el ámbito internacional. Sin duda alguna, Ensayo es difícil de ubicar como perteneciente a una escuela psicológica en particular. Autores tan variados como los psicoanalistas Sigmund Freud y Carl Gustav Jung; así cómo Karl Koffka, uno de los fundadores de la psicología Gestalt, y filósofos tan opuestos como el antinazi Max Scheler y el fascista Giovanni Gentile, se encuentran citados en el libro. La obra de Chávez carece de aportaciones de carácter científico realizadas por él mismo. Sin embargo, su riqueza recae en las numerosas reflexiones personales sobre la situación que privaba en los adolescentes mexicanos y el estado de la pedagogía nacional durante la década de los veinte del siglo xx.

Uno de los objetivos centrales de este artículo ha sido responder por qué Chávez se interesó en escribir un libro enfocado principalmente en los adolescentes. Parcialmente esta pregunta ha sido contestada en las primeras páginas de este artículo. No obstante, podemos encontrar más respuestas a dicho cuestionamiento en las primeras páginas de Ensayo.

Para Chávez, la Primera Guerra Mundial había representado el punto cumbre de la "anarquía moral" que privaba en el mundo, de igual forma veía con inquietud los conflictos bélicos que se estaban generando entre las naciones y al interior de las mismas. La atención del mexicano se enfocó en la restitución de los valores occidentales a partir de una apropiada formación de los adolescentes, quienes por sus características moldeables y su cercanía con la edad adulta, podían contribuir a la configuración de un nuevo panorama nacional e internacional a corto plazo. Esta misión se centraba en la educación y en los educadores:

por lo cual urge rehacer la cultura, restableciendo la trabazón lógica, psíquica y social del mundo, caso de que sea posible hacerla, o forjar, en último y desesperado extremo, una nueva cultura. Mas como la inmensa labor que tal propósito y semejante necesidad suponen no puede realizarse sino por las generaciones que están llegando, y que tienen que ser guiadas por las que ahora le preceden. [...] Más aún, sobre quienes tienen a su 
cargo la educación de los adolescentes: de una parte, porque los adolescentes van a entrar muy pronto en la vida pública, y puede hacer cada día más incierta su marcha, o, por el contrario, encauzarla; de otra, porque en la adolescencia llega a su máximo el peligro de extraviarse, separándose de toda organización y norma. ${ }^{19}$

Para Chávez sólo se podía construir una tipología de la adolescencia partiendo de un análisis comparativo con otras etapas de la vida humana, como eran la niñez y la adultez. Aunque era consciente de una amplia gama de clasificaciones por edad y de una forma individualizada de maduración psíquica, mental, educativa y física en cada ser humano, en Ensayo ofrece una propuesta bastante flexible. De manera generalizada nuestro autor bosqueja seis edades básicas: niñez, juventud, edad viril, edad madura, senectud y decrepitud, pero centra su atención en las dos primeras. ${ }^{20}$ Subdivide a la infancia en tres partes: "pre-escolar o extrema infancia, edad de párvulos y puericia", que abarcarían desde el nacimiento hasta "los diez, once, doce o trece años y rara vez los primeros catorce, quince o dieciséis". ${ }^{21}$

El segundo periodo de la vida correspondía a la juventud que era subdividida en "adolescencia y segunda parte de la juventud". La primera iniciaba a "los nueve ańos y medio, diez, once, doce, trece o catorce años y concluye hasta los diez y ocho o veintitrés [sic] o, en algunos casos, a los veinticuatro o los veinticinco años". ${ }^{22}$ Ésta a su vez podía ser seccionada en un periodo de "aceleración inicial"; en uno posterior denominado de "aparición ostensible de la pubertad y desarrollo de la misma"; para concluir en la llamada "post-adolescencia inmediata, primera juventud o estabilización de la pubertad". La segunda parte de la juventud comenzaba a los veintiuno o veinticinco y se alargaba hasta los treinta ańos, se le consideraba como un periodo de estabilización en los aspectos físicos, psíquicos

${ }^{19}$ Ezequiel A. Chávez, Ensayo, 13.

${ }^{20}$ Para la edad viril, el autor no hace anotación alguna si dentro de esta periodización estaban incluidas las mujeres o cuál podría ser la denominación correspondiente para el género femenino. Ibid., 20.

${ }^{21}$ Ibid., 19.

${ }^{22}$ Ibid., 22. 
y sociales. Estos criterios de subdivisión fueron construidos a partir de la capacidad y perfeccionamiento del lenguaje durante la nińez y del desarrollo físico acelerado iniciado en la pubertad. Sin embargo, a lo largo del libro otros elementos constitutivos del ser humano un poco más especializados como la psique, el comportamiento y la conducta fueron incorporándose a la construcción del adolescente $y$, por lo tanto, a su diferenciación con respecto al nińo.

El psicólogo mexicano afirmaba, intentando resaltar la importancia de prestar atención a las habilidades y características de cada una de las edades, que en muchos casos los redactores de ciertas legislaciones se habían percatado empíricamente de la presencia de ciertas cualidades particulares de los individuos de diferentes edades. Tal era el caso que se veía reflejado en los estatutos plasmados en el Código Penal Mexicano en relación con la responsabilidad criminal hasta los catorce años; la necesidad de tutoría de los menores de dieciocho ańos, y la adquisición de la ciudadanía a los veintiún años en hombres solteros y dieciocho en casados. Así mismo, mencionaba al Código Civil del Distrito Federal en el que se prohibía el matrimonio a los hombres menores de catorce años y a las mujeres menores de doce; y a la emancipación femenina de la casa de sus tutores hasta los treinta ańos de edad, a pesar de haber alcanzado ya la mayoría de edad. ${ }^{23}$

Heredero del idealismo alemán que tendría repercusión en la metodología científica desde principios del siglo XIX, el vitalismo fisiológico se convirtió en una hipótesis que imperó en las explicaciones que Chávez realizó sobre el "repentino" inicio de la pubertad y de la adolescencia en el ser humano. La idea fundamental de esta doctrina fue la creencia en una fuerza vital rectora de la vida y del funcionamiento íntimo de los seres vivos. ${ }^{24}$ Basado en el elan vital del filósofo francés Henri Bergson, Chávez basó gran parte de sus

${ }^{23}$ Ibid., 24-25.

${ }^{24}$ Esta filosofía entró con fuerza en Francia, algunos de sus representantes más importantes fueron François Boissier, Philippe Pinel, Theophile Bordeu, Xavier Bichat y Paul Joseph Barthez, sin embargo, fue desacreditada por muchos científicos en 1928 tras unos experimentos realizados por el pedagogo y químico alemán Friedrich Wöhler. Josep Lluis Barona, Historia de la ciencia y de la técnica, núm. 46 (Madrid: Akal, 1991), 38. 
postulados en esta noción, ostentándola como una súbita energía causante del despertar de los caracteres y de las funciones fisiológicas de los niños y adolescentes:

Llega luego otra vez rítmicamente, y titubeando, abre las puertas de la juventud, la edad de más numerosas y variadas crisis, la adolescencia, que entra primero en su periodo de aceleración inicial, arrebatada y a la carrera, por dos años, con su crecimiento súbito del organismo, a lo menos en gran número de casos estirándose y alargándose todo; que luego, muestra, casi todos juntos, los signos materiales y externos de la pubertad, apuntándolos al principio, desmañadamente, en un breve periodo de cinco a siete meses, y reiterándolos después con firmeza, en el siguiente año y medio, con lo cual acaba la edad sin gracia, y se pasa a la edad de la plenitud posterior. ${ }^{25}$

De forma exhaustiva y profunda Chávez desarrolló una explicación sobre cada uno de los sistemas y aparatos del cuerpo humano. Particularmente se concentró en los cambios que sucedían durante la adolescencia y las repercusiones del "mal funcionamiento" de sus órganos, de las secreciones (las "hormones" conocidas hoy en día como hormonas) y fluidos (específicamente la sangre). El deficiente seguimiento de las recomendaciones médicas (profilaxis) podía propiciar un mal desarrollo del adolescente que repercutiría en su vida futura, en el progreso social y en el mejoramiento nacional. A este respecto, Chávez alertó a sus lectores sobre la susceptibilidad de esa edad a adquirir distrofia y enfermedades, promovidas por el esfuerzo físico del crecimiento: "Todo el mundo sabe que entonces la resistencia física disminuye, y que todo el cuerpo presenta menos inmunidad contra las influencias morbíficas $[$ sic $]$ ". ${ }^{26}$

Un ejemplo que ilustra dicho escenario es el funcionamiento del aparato circulatorio y su relación con el crecimiento muscular. Algunos médicos opinaron que el desarrollo desequilibrado de los múscu-

${ }^{25}$ Ezequiel A. Chávez, Ensayo, 28.

${ }^{26} \mathrm{Ibid}$., 54. Me resulta interesante la pervivencia de algunos elementos de la teoría humoral. La influencia morbífica es uno de ellos y se entiende como "Las potencias enemigas, tanto físicas como morales que afectan nuestras vidas". Fernando Rizquez, Psiquiatría y homeopatía (Nueva Dehli: B. Jain Publishers, 2005), 159. 
los podía afectar al corazón, cuyo proceso de crecimiento no era tan vertiginoso. Las actividades como el atletismo promovían mayor trabajo cardiaco y por lo tanto el riesgo de contraer hipertrofia:

proscribir entre los adolescentes, el boxeo, las luchas, el pugilato y aún más las formas violentas de football, deportes, atléticos y "campeonatos", que no sean más que supervivencia de otras edades y descompensen, irremediablemente a los que a ellos se entreguen y sustituirlos por juegos deportivos, acompañados y rectificados por acertados ejercicios respiratorios, $\mathrm{y}$ por los que, sin violencia, fijen las actividades correctas y hagan que el organismo funcione de modo armonioso. ${ }^{27}$

La mayoría de los ejercicios estaban contraindicados para las adolescentes. Chávez afirmaba, basado en algunos reportes de la Board of Education de Londres, que las actividades ideales para las jovencitas eran caminar, coser y jugar tenis. El objetivo principal era reservar las energías que en un futuro se utilizarían durante el embarazo. La diferenciación entre hombres y mujeres no sólo era ostensible a partir de las recomendaciones de las actividades físicas que debían realizar y en la fisiología de sus cuerpos, sino en la enseñanza de labores propias para cada género y en los comportamientos que debían seguir. ${ }^{28}$

Otra inquietud se centró en las relaciones entre ambos sexos. Chávez veía con desagrado que en algunas naciones modernas se intentara borrar la diferenciación de géneros. De hecho, la concebía como un signo más de la "degeneración social" que él presenciaba. Para Chávez, las féminas eran las garantes de la sobrevivencia de las estructuras familiares y, por lo tanto, debían ser protegidas e inclusive sacralizadas:

Por eso los adolescentes necesitan considerar siempre, como divina casi inaccesible e intangible, a la mujer, para que de veras la amen; y por eso necesitan no ignorar ellas, como dice Muriac, "lo que ganaba su cuerpo,

${ }^{27}$ Ibid., 28.

${ }^{28}$ Ibid., 109. 
cuando era inaccesible"; no sólo para ser amadas, sino para que los adolescentes las amen, conserven incólume y acrediten su amor, por el que unos y otras podrán ser lo mejor de cuanto mejor puedan ser. ${ }^{29}$

Aunque su opción predilecta fue la separación de los contenidos de la enseńanza dependiendo del género del educando, Chávez no se opuso a la posibilidad de la coeducación, pues, consideraba saludable la convivencia natural entre chicos y chicas. ${ }^{30}$

El control del instinto sexual que surgía durante la adolescencia - pensaba - no guardaba concordancia con los fenómenos psíquicos de aquella edad. Por lo tanto, los jóvenes por naturaleza, no deberían tener interés por mantener "relaciones físicas" con las mujeres. Chávez corroboraba esta teoría aludiendo al "natural recato" de los adolescentes. Para él, la prensa, el cine y los espectáculos teatrales eran los responsables de terminar con el "misterio femenino" y propiciar que la curiosidad de los jóvenes se elevara a otros niveles más pecaminosos:

Las excitaciones de la vida moderna, las desnudeces, las sugestiones picantes, las obsesiones son tan numerosas, se multiplican a tal grado, se vuelven tan transparentes, que muchos de los niños no pueden ya resistirlas, porque para ellos desaparece el prestigio del otro sexo, y el de su misterio, a la vez que la coacción social que normalmente este $[s i c]$ lleva implícito, y, si no siempre de hecho, si, al menos, en pensamiento, pasan a la realización de actos de consecuencias gravísimas, con lo que llegan a sufrir una especie de contractura mental, un traumatismo psíquico. ${ }^{31}$

${ }^{29}$ Para Chávez el cristianismo dotó a la mujer de enaltecimiento, sobre todo, tomando como ejemplo y objeto de culto a la virgen María. Ezequiel A. Chávez, Ensayo, 279.

${ }^{30}$ En aquellos años existía una diferencia entre la coeducación y la educación mixta. En la coeducación niños y nińas asistían a un mismo plantel educativo, pero se les enseñaban labores y conocimientos acordes a su edad y género. La educación mixta, por su lado, era la enseñanza a hombres y mujeres de las mismas materias con los mismos contenidos. Algunos ejemplos sobre este tipo de sistemas en Elsie Rockwell, Hacer escuela, hacer estado. La educación posrevolucionaria vista desde Tlaxcala (México: El Colegio de Michoacán, Ciesas, Cinvestav, 2007), 307-311.

${ }^{31}$ Ezequiel A. Chávez, Ensayo, 176-177. 
La adolescencia fue considerada como un periodo de vida donde el proceso de razonamiento y de la elaboración de juicios se encontraba en proceso de desarrollo. Era menester de los educadores y de los padres de familia ser capaces de orientar a los adolescentes por el camino correcto mediante la educación moral y el entrenamiento del sentido del juicio. De igual importancia fue la preparación del docente para detectar cualquier anormalidad en el desarrollo físico y psicológico de sus pupilos. De ahí que el libro estuviera dedicado principalmente a los profesores normalistas quienes podían dar seguimiento al desarrollo de sus alumnos.

Otro factor determinante en la "conducta" de los jóvenes fue el medio social. La situación política del país, es decir, la Revolución mexicana y la llegada al poder de las diferentes fracciones se había convertido en un elemento perturbador que ponía en riesgo la conducta "normal" de los adolescentes. De hecho, Chávez externaba su preocupación afirmando que las condiciones de animadversión entre los diferentes sectores políticos y religiosos habían propiciado un ambiente de neurosis dentro de la población mexicana. Esta situación no había permitido la constitución de un medio propicio para el desarrollo de los más jóvenes. ${ }^{32} \mathrm{La}$ adolescencia era una edad en la que los sujetos corrían el peligro de ser manipulados debido a su sensibilidad, a su tendencia a "exagerar" y a la facilidad con que eran impresionados por "agitadores de profesión". Para el autor era obligación del Estado mantener la buena salud de sus gobernados y ofrecer escuelas bien organizadas que tuvieran como uno de sus objetivos construir hombres cultos que difícilmente cayeran en este tipo de supuestos alborotos.

Una de las primeras medidas que Chávez propuso para sobrellevar esta situación fue la impartición de la educación moral a la par

\footnotetext{
${ }^{32}$ Chávez recogió algunas ideas del psicólogo inglés R. G. Gordon para interpretar el caso mexicano: "el mismo Gordon sustenta, cuando dice que estar totalmente libre de neurosis entrańa tener en cada momento perfecta armonía, dentro de la propia personalidad y perfecta adaptación mental a las circunstancias del medio ambiente, condiciones casi imposibles de cumplir en sociedades cómo México, constantemente divididas por hondas animadversiones de tendencias políticas y de frenéticos egoísmos, que se perpetúan, como sus seculares e inveterados conflictos religiosos, al través de toda su historia [sic]”. Ezequiel A. Chávez, Ensayo, 88.
} 
de la enseñanza de la historia y la geografía. ${ }^{33}$ Chávez daba cuenta, a lo largo de las páginas de Ensayo, de la forma en que el grupo triunfante de la Revolución había reinterpretado la historia del porfirismo, olvidando el progreso nacional alcanzado durante aquella época, el esfuerzo educativo y un proyecto nacionalista ya desde entonces presente:

Patentizan lo justificado de estos asertos las actitudes mentales que toman los adolescentes en épocas de grave conmoción general: la que se ha llamado a si propia, en México, la generación de 1915 [...]. Cuando volvieron un poco en sí, los adolescentes de esa generación que así sentían tuvieron fuerzas para dirigir la mirada en torno suyo, y vieron los colores del cielo y la tierra [...] Casi todos apasionados, les parece que ante ellos México nacía; que nunca había existido antes; que estaba creándose a sí mismo; que cuando lo había precedido no había sido más que una horrible pesadilla. [...] Esto explica que un joven orador del Congreso de Maestros efectuado en 1928 en la ciudad de México declaró impetuosamente, en la tribuna, que México no había existido antes de 1910, que de entonces a acá databan las canciones mexicanas, la poesía mexicana, la novela mexicana, el arte mexicano [...] Sólo que en su descubrimiento de que México existiera, los nuevos mexicanos, adolescentes de entonces, proyectada su vida desde ellos, no pudieron reconocer ningún valor espiritual anterior, y no viéndolo, les paso lo que al niño que cierra los ojos y, sumergido en tinieblas, no ve la luz. ${ }^{34}$

Esta crítica realizada contra la llamada Generación de 1915 es un ejemplo de la aplicación de las teorías psicológicas en la interpretación de los escritores y burócratas de la "nueva historia nacional" postrevolucionaria. ${ }^{35}$ Este modelo fue utilizado por nuestro autor

${ }^{33}$ La inclusión o no de dichas materias junto con otras disciplinas humanísticas como la filosofía y la literatura, habían sido objeto de discusión en los congresos educativos mexicanos donde se debatieron y elaboraron los planes de estudio de la Escuela $\mathrm{Na}$ cional Preparatoria desde finales del siglo XIx. Charles Hale, La transformación del liberalismo en México, 221-265.

${ }^{34}$ Ezequiel A. Chávez, Ensayo, 427-428.

${ }^{35} \mathrm{La}$ Generación de 1915 estuvo formada por Antonio Castro Leal, Alberto Vázquez del Mercado, Vicente Lombardo Toledano, Teófilo Olea y Leyva, Alfonso Caso, Manuel Gómez Morín, Jesús Moreno Baca, Daniel Cosío Villegas, Miguel Palacios Macedo y 
como una herramienta para desacreditar los discursos de ciertos funcionarios “jóvenes”. A su vez, contribuyó a que Chávez legitimara su permanencia en las instituciones posrevolucionarias por su experiencia administrativa, académica y de vida sobre la de los miembros de dicha generación que se habían integrado al "nuevo" proyecto nacional. ${ }^{36}$

Las observaciones de nuestro autor no sólo se dirigieron a mandos medios en el poder, sino que inclusive alcanzaron a la figura presidencial. Como devoto seguidor del catolicismo y defensor de la educación moral, Chávez atacó la expedición de la Ley reglamentaria del articulo 130 Constitucional por parte del presidente en turno Plutarco Elías Calles, decretada en $1924 .{ }^{37}$ Su lucha por la libertad educativa también podía observarse en su defensa por la autonomía de la Universidad Nacional de México. ${ }^{38}$

El presidente Calles compartió con Chávez la preocupación por implantar, dentro de la enseñanza, la educación moral en los planteles educativos. El secretario de Educación Pública en turno, José Manuel Puig Casauranc, durante un acto organizado por la Asociación Católica de Jóvenes Mexicanos en 1926, había mencionado las labores del gobierno realizada en las escuelas:

Narciso Bassols. Esta Sociedad de Conferencias tuvo como origen el estallido de la revuelta revolucionaria. Su objetivo fue aprovechar la apertura de los nuevos regímenes revolucionarios a la participación juvenil en la reconstrucción nacional y contribuir a ella desde la vía intelectual. Enrique Krauze, Caudillos culturales en la Revolución Mexicana (México: Siglo XXI editores, 2000), 11-13.

${ }^{36}$ Chávez había sido llamado a participar en varios proyectos con miembros de dicha generación. Durante el gobierno de Huerta, en 1913, había impulsado junto con Nemesio Naranjo una reforma antipositivista en la Universidad Nacional. Para concretar dicha modificación se había convocado a un Consejo en el que había participado Antonio Caso. Alicia Meyer, coord., México en tres momentos: 1810-1910-2010, vol. 2 (México, IIH-UNAM, 2007), 350; Narciso Bassols, en su cargo como secretario de Educación Pública (1931-1934) impulsó el laicismo en la educación y la educación sexual contraviniendo la postura de Chávez quien votó en contra y en pro de la educación religiosa. Alberto Saladino García, Humanismo mexicano del siglo XX, vol. I (Toluca: Universidad Autónoma del Estado de México, 2004), 37.

${ }^{37}$ María Alicia Puente Lutteroth, Movimiento cristero: una pluralidad desconocida (México: Editorial Progreso, 2002), 92-93.

${ }^{38}$ En 1914, presentó un proyecto de ley de independencia de la Universidad Nacional. Véase Javier Mendoza Rojas, Los conflictos de la UNAM en el siglo XX (México: UNAM, CESU, 2001), 56. 
En las escuelas primarias, aparte de la instrucción que se procura y junto con la formación de carácter y de sentimientos que nosotros procuramos conseguir por el Código de Moralidad a que aludía, en las escuelas primarias, digo, debe nacer, debe incubarse el concepto de Patria. ${ }^{39}$

La gran diferencia entre las preocupaciones de Puig y Chávez fue el papel de los maestros en la enseńanza religiosa dentro de los planteles educativos. Para Puig, la enseñanza de la religión no había sido prohibida sino confinada al ámbito del hogar a cargo de los padres de familia, pues, los "sacerdotes y maestros podían mezclar en sus enseñanzas de naturaleza religiosa venenos y toxinas de naturaleza política y social". ${ }^{40}$

Chávez también abogaba por la participación activa de los progenitores en la formación de sus hijos adolescentes. A diferencia de Puig afirmaba que las "religiones más puras llevan en sí, sentimientos morales, un sistema de reglas de conducta individual y social, anhelos de perfección y de servicio [...] y su impartición era imprescindible, por lo menos en las escuelas particulares". ${ }^{41}$ Seguramente a partir de dicha reflexión es que podemos explicar la extensa obra que Chávez escribió en torno a personajes y temas de la historia de México relacionados con el ámbito religioso. ${ }^{42}$ Tanto la religión, como la historia y la filosofía proveían de ejemplos constructivos

${ }^{39}$ Secretaría de Educación Pública, El esfuerzo educativo en México (México: SEP, 1928), LXXVI-LXXVII. La historiadora Engracia Loyo apunta que la primera versión del Código de Moralidad fue elaborada en Cuba, pero la versión mexicana fue una traducción de una edición estadounidense. Por otro lado, Loyo también afirma que el presidente Calles aprovechó el conflicto con la Iglesia para presentarse como el defensor de la moralidad cívica pública, buscando que el ideal del buen mexicano coincidiera con la del buen cristiano. Engracia Loyo, Gobiernos revolucionarios y educación popular en México, 1911-1928 (México: El Colegio de México, 1999), 252.

${ }^{40}$ Secretaría de Educación Pública, El esfuerzo educativo en México, Lxxvi.

${ }^{41}$ Ezequiel A. Chávez, Ensayo, 377.

${ }^{42}$ Puedo mencionar algunas obras como Sor Juana Inés de la Cruz: ensayo de psicología y de estimación del sentido de su obra y de su vida para la historia de la cultura y de la formación de México (1931); El primero de los grandes educadores de la América: fray Pedro de Gante (1934); La evangelización de los indios (1956); y otras obras que resaltaban los valores cívicos: Hidalgo (1956), Morelos (1957), Agustín de Iturbide: libertador de México (1957), entre otras. 
para los adolescentes, por ello es que para nuestro autor estas materias eran indispensables para una óptima formación adolescente.

Una de las premisas básicas que Chávez propuso en su libro fue la adaptación de los trabajos intelectuales a las condiciones mentales de los educandos. El objetivo de la educación fue el "perfeccionamiento del individuo con relación a la sociedad". ${ }^{43}$ Nuestro autor rescató de Platón la idea de que en el mundo existían individuos que "llevaban en sí la posibilidad de iniciar transformaciones", y otros que estaban "destinados a repetir lo que ya se había venido haciendo". ${ }^{44}$ Chávez afirmaba que esta desproporción garantizaba la sobrevivencia de los pueblos:

Un pueblo que sólo tuviera hombres inquietos, de desequilibrado espíritu, superiores tal vez en un sentido y aún geniales, podría estár condenado a extinguirse rápidamente, porque le faltara la fuerza poderosa de la multitud, conservadora y lenta, que regenera y mantiene las energías biológicas de la humanidad, y así asegura la posibilidad del advenimiento de los hombres superiores que puedan guiarle. ${ }^{45}$

En algunos estudios científicos de la época, el estado ideal del individuo era la correspondencia entre las edades cronológica, anatómica, fisiológica, psicológica, educativa y social. Chávez centró su atención en la correlación de la inteligencia con la edad cronológica. Basó sus observaciones en las llamadas "medidas de habilidad mental cognitiva”, a partir de una metodología formulada por Alfredo Binet para estudiar los niveles de inteligencia de algunos reclutas en los Estados Unidos durante la Gran Guerra. Aunque sin realizar un estudio tan exhaustivo del caso mexicano y más bien basándose en sus observaciones sobre la población indígena, Chávez concluyó que entre la mayoría de los habitantes de ambas naciones persistía un desfase entre la correspondencia de las diversas edades y su capacidad cognitiva. Dicha situación convertía a la mayoría de la población en "mentalmente inferior".

${ }^{43}$ Ezequiel A. Chávez, Ensayo, 408.

${ }^{44}$ Ibid., 121.

${ }^{45}$ Ibid., 122. 
Chávez alegaba que en México, donde la mayoría de la población era indígena, las malas condiciones físicas, la miseria y el desamparo milenario habían contribuido al deficiente desarrollo intelectual, mental y físico. Se preguntaba insistentemente si podría considerar a la raza indígena de aquellos ańos, como mentalmente inferior con respecto a otros grupos raciales. Y si esa deficiencia había empezado desde su pubertad.

Una de las propuestas educativas en Ensayo fue la adaptación de la educación a las capacidades del educando para que de esta forma pudiera ajustarse a su medio social:

Defendámonos, sin embargo, contra el pesimismo: el mismo Góddard nos lo advierte: si se educa atinadamente a quienes parezca que no pueden bastarse a sí propios; al morón, aún al imbécil, y al que se tacha de idiota, adquirirán aptitudes suficientes, para algunos trabajos manuales, y podrán afrontar las dificultades de la existencia, con buen ánimo: alegre y servicial. ${ }^{46}$

Una educación inadecuada, tanto para débiles mentales como para los adolescentes, era concebida como un peligro inminente para la sociedad. Un individuo descorazonado por no poder alcanzar lo que la educación le imponía podría convertirse en un ser "agriado" capaz de cometer un crimen o en un "agitador" que pudiera provocar tormentas sociales. Para evitar dicha situación, Chávez recomendaba la elaboración de programas especiales de estudios para "morones" de ocho a doce años de edad mental haciendo hincapié en los trabajos manuales y la educación industrial. Recomendaba, la exclusión casi completa de la enseñanza abstracta. ${ }^{47}$

Chávez alertaba una y otra vez sobre la imposibilidad de someter a los adolescentes a una definición única y, por lo tanto, a programas generalizadores de educación. La adolescencia era una edad vinculada con el nacimiento de la individualidad. ${ }^{48}$ Observaba una peque-

${ }^{46}$ Ibid., 118-119.

${ }^{47}$ Ibid., 126.

${ }^{48}$ Algunas de las recomendaciones que Chávez realizó y en las que fue recalcitrante: "Que cada adolescente es un problema sui géneris, como lo es cada uno de los educandos, como lo es cada uno de los hombres: que unos hay, en quienes tal o cual tendencia 
ña diferenciación en el desarrollo físico de los adolescentes de las zonas rurales con respecto a los citadinos: la pubertad aparecía en promedio a los trece ańos y siete meses y medio en el campo y trece años y nueve meses en las ciudades. ${ }^{49}$

La formación del instinto gregario en los adolescentes fue un tema al que Chávez prestó mucha atención. Sus apreciaciones y recomendaciones pueden encontrarse en una cantidad bastante representativa de parágrafos a lo largo del Ensayo. El fenómeno de la congregación de adolescentes fue una característica que la psicología definió como propia de esa edad:

Así los veis esperarse, llamarse, abatirse sobre las bancas del Luxemburgo como gorriones; amontonarse en las cervecerías en los cafés. No tienen todavía vida individual [...] Aún para preparar un concurso les gusta ser varios. Y si no fuera más que para preparar un concurso!... [sic] Su noctambulismo les viene de esta repugnancia a volverse a encontrar solos entre cuatro paredes. Por lo mismo, se acompańan indefinidamente unos a otros y vuelven y revuelven sobre sus pasos, hasta que el exceso de fatiga los obliga, por fin a irse a dormir. Como en píos la vida de los gorriones, en conversar se pasa la de los adolescentes. ${ }^{50}$

Sería incorrecto plantear que la idea de congregar jóvenes en organizaciones con fines educativos y deportivos, como los boy scouts, sólo haya ocurrido durante el siglo xx. Esta tradición puede ser rastreada inclusive en la Grecia clásica y en el mundo prehispánico. ${ }^{51}$

instintiva predomina tanto, que oscurece los demás rasgos característicos; otros, en que quienes una o varias de estas tendencias, faltan del todo, o casi del todo; ya, en éste, aparecen como indestructibles ciertos hábitos o vicios sociales: el disimulo, quizás; la hipocresía, la cobardía, la adulación, la bajeza [...], que en parte originan reiterados antecedentes históricos, o pertinaces condiciones de determinado medio ambiente social; ya en aquel, se revelan sólo influencias perniciosas menos generalizadas: características unas de ciertas razas, de determinado grado de falta de cultura”. Ibid., 410.

${ }^{49}$ Ibid., 125.

${ }^{50}$ Pasaje rescatado por Chávez de François Mauriac, La Jeune Homme (París: éd. Hachette, 1926). Ibid., 285-286.

${ }^{51}$ Véase Giovanni Levi y Jean Claude Schmitt, Historia de los jóvenes, 2 vols. (Madrid: Taurus, 1996). Podemos ejemplificar el caso prehispánico mexicano con las dos instituciones educativas más estudiadas de aquel periodo histórico: el calmecac adonde 
Sin embargo, fue a finales del siglo XIx y durante las primeras décadas del xx que el modelo corporativista fue el predominante en la construcción de instituciones y organizaciones juveniles, organizadas o custodiadas por diferentes sectores adultos (gobiernos, líderes religiosos, padres de familia, maestros, partidos políticos, entre otros) y estudiantiles. ${ }^{52}$

La escuela para nuestro autor parecía una institución capaz de homogeneizar el comportamiento y la moda en el vestir y en el hablar de los adolescentes. Chávez observó con preocupación que el maltrato a los maestros y a los mismos compañeros era arremedado grupalmente por los estudiantes preparatorianos. Sin embargo, era optimista ya que veía a los planteles educativos como un medio en el que se podía promover algún otro tipo de "contagio psico-fisiológico" que de manera más positiva contribuiría a la formación de lazos de compańerismo y de ayuda humanitaria. ${ }^{53}$

Debido a que esta tendencia gregaria les era innata, al igual que la propensión al desorden, era necesario el control y la organización de actividades grupales desde el ámbito escolar. ${ }^{54}$ Uno de los primeros objetivos era la formación de ideales de pertenencia bajo los cuales los adolescentes se pudieran identificar y congregar. Al respecto Chávez citaba el caso de la construcción del concepto y de las tradiciones ligadas a la noción de Alma Mater en las universidades ingle-

eran enviados los hijos de los nobles; y el telpochcalli, destinado a los hijos del resto de la población. Alfredo López Austin, Educación mexica. Antología de textos sahaguntinos (México: IIA-UnAm), 1985.

${ }^{52}$ Durante la primera década del siglo xx se formó una extensa red institucional de partidos, sindicatos, organizaciones campesinas y ligas. Fue durante la década de los treinta cuando el corporativismo se consolidó convirtiéndose en una de las principales formas de interlocución admitidas entre los sectores sociales y el gobierno. Beatriz Urías Horcasitas, Historias secretas del racismo en México (1920-1950) (México: Tusquets, 2007), 18.

${ }^{53}$ De hecho las observaciones de nuestro autor se centraban en la formación de clubes, sociedades, asociaciones de diversas especies para fines escolares, por diversión, para realizar excursiones e inclusive para desarrollar actividades cívicas. Ezequiel A. Chávez, Ensayo, 228.

${ }^{54}$ Como ya pudimos observar, Chávez apelaba a que en las labores escolares colectivas no se pusiera a competir a las mujeres. Y proponía el establecimiento de ejercicios de cooperación, coordinación y servicio social. Ibid., 125. 
sas. ${ }^{55}$ Esta reflexión nos lleva a pensar en la posibilidad de que Chávez tuviera cierta intervención en la construcción de narrativas, símbolos y actividades similares en la Universidad Nacional de México.

En los Estados Unidos, desde mediados del siglo xIx, la idea de agrupar adolescentes en organizaciones controladas por los adultos, según algunos historiadores, partió de que niños y jóvenes citadinos se congregaban en pandillas o grupos delictivos. ${ }^{56}$ La gran proliferación de estas comunidades de adolescentes propiciaron que la burguesía citadina incitara a los reformistas estadounidenses y a las legislaciones regionales a prestar atención sobre lo que consideraban una patología social, un peligro para la salud pública y una amenaza para la vida familiar. Los sistemas legal y judicial de la época fueron modificados para contener, corregir e integrar a los adolescentes dentro del ámbito penal. ${ }^{57}$

En México, según José Ángel Ceniceros y Luis Garrido, el Código Penal de 1871 había deslindado de responsabilidad delictiva a los niños menores de nueve años. Mientras que para la resolución de la culpabilidad o no de los jóvenes de entre nueve y catorce años se permitió un dictamen pericial para dictar sentencia y responsabilidad en el delito por el cual habían sido incriminados. ${ }^{58}$ Dicho Código fue modificado constantemente tomando en consideración las observaciones del sistema penitenciario del estado de Nueva York en los Estados Unidos con respecto al tratamiento de los adolescentes. Fue hasta 1928 cuando en México se establecieron los Tribunales para Menores. Aunque no pretendo ahondar en dicho tema en este

55 Nuestro autor afirmaba que en las universidades inglesas se habían formado grupos de solidaridad juvenil conforme a los intereses y aspiraciones compartidas. La construcción del Alma Mater entonces servía como una forma organizada de suscripción para alcanzar dichos objetivos. Ibid., 65.

${ }^{56}$ Puedo mencionar por ejemplo a Jon Savage, Teenage. The Creation of Youth Culture (Londres: Viking Penguin, 2007); y Timothy J. Gilfoyle, "Street-rats and Gutter-snipes: Child Pickpockets and Street Culture in New York City, 1850-1900, Journal of Social History 37(4) (verano 2004): 853-882.

${ }^{57}$ Timothy J. Gilfoyle, "Street-rats and Gutter-snipes", 853.

${ }^{58}$ José Ángel Ceniceros y Luis Garrido, La delincuencia infantil en México (México: Ediciones Botas, 1936), 18. 
artículo, llama mi atención la concordancia entre el "redescubrimiento" del fenómeno de la adolescencia y las siguientes reflexiones realizadas por Chávez en torno a los factores que impulsaban a los adolescentes a delinquir y la proliferación de libros sobre el proceso penal en los menores de edad. ${ }^{59}$

En algunos parágrafos de Ensayo, Chávez aludía tanto a los factores sociales como a los fisiológicos, que creyó influían en los adolescentes para la perpetración de actividades delictivas y la adopción de vicios. Chávez sugería que un adolescente se convertía en delincuente debido al "descuido social" y al incorrecto establecimiento de programas educativos que ignoraban el desarrollo armónico del joven. Por su parte, la propensión a caer en el vicio guardaba correspondencia con las patologías de las glándulas suprarrenales que a su vez eran las responsables de los cambios en el temperamento, sobre todo, en el desarrollo del instinto belicoso. ${ }^{60}$ Fue por lo tanto inconsistente para él la noción de castigo para los menores, razón por la que abogó por programas de regeneración y readaptación a la sociedad. ${ }^{61}$

Para Chávez la rebeldía era otra característica propia de la adolescencia. Era en esta etapa, la última de formación fisiológica y la primera de gran desarrollo muscular e incremento de la fuerza física, que motivaba al joven a compararse con los nińos y los adultos para comprenderse como un ser independiente. La tarea del maestro era encauzar la rebeldía por medio de la enseñanza del civismo, mientras que el cometido de la sociedad era crear sociedades venerables, leyes justas y un sistema judicial que hiciera respetar la legislación. ${ }^{62}$

${ }^{59}$ Podemos encontrar obras como la de Manuel Velázquez Andrade, La delincuencia juvenil, México, Editorial Cultura, 1932; Alfonso Quiróz Cuarón, Cursos sobre delincuencia infantily juvenil, 1938-1938, México, s.e. Una posible respuesta a dicha diferencia es el proceso de urbanización y modernización nacional en México, sobre todo, en el periodo posterior a la Revolución mexicana.

${ }^{60}$ Ezequiel A. Chávez, Ensayo, 1956, 96.

${ }^{61}$ Chávez apunta que una de las causas del crimen era la mal encaminada imaginación de los adolescentes. Ésta, afirmaba nuestro autor, se debía a la mala formación educativa del joven en el desarrollo del sentido del juicio sobre el de la acción impulsiva. Ibid., 406-409.

${ }^{62}$ Ibid., 342. 
Como un tema de conflicto intergeneracional presente en todas las épocas, la moda fue un tema de reparo para nuestro autor. Estrechamente ligada con la cuestión del desarrollo de los caracteres sexuales durante la pubertad, la moda según Chávez fue una forma de asimilación de los jóvenes de los cambios fisiológicos de sus propios cuerpos. Las transformaciones podían observarse en el cambio de las posturas, en el modo de andar, de correr y hasta en aspectos más materiales como su indumentaria: longitud, anchura, corte y color de las ropas. Las nuevas formas de sociabilización de los adolescentes, los cambios fisiológicos y la creciente cultura material enfocada en este grupo de edad promovieron una nueva gama de pensamientos y comportamientos entre los jóvenes:

Incesantes sorpresas; inagotables preguntas que no llegan a formularse; $y$ envidia, porque no se tiene lo que en los demás se admira; admiraciones furtivas o descaradas; hondas ensońaciones posteriores. Con todo ello el alma se centra; se aguza y languidece; se estira; se lanza y sobre sí propia se refleja. Adquiérese una aguda habilidad para percibir cuanto a individuos del otro sexo se refiere. Los criminalistas fundan en ella el valor excepcional de los testimonios de los adolescentes a este respecto, y más lo conceden a los de las adolescentes. ${ }^{63}$

Chávez observó con desesperanza que las nuevas generaciones ya no vestían con modestia y que el recato característico de los años de la juventud de nuestro autor prácticamente se encontraba olvidado. Parafraseando a George Dumas afirmaba que la búsqueda del "placer por el placer mismo o por el libertinaje" era el objetivo de los adolescentes de finales de la década de los veinte. Una aparente revolución sexual parecía incomodar a nuestro autor, quien manifestaba su preocupación por la presencia de lo que interpretaba como una invasión de novelas, literatura, arte y teatro con un alto contenido sexual. El baile, bajo los ritmos del charleston y del jazz, fue una de las actividades sociales que desconcertaron a Chávez con mayor ímpetu, pues, afirmaba que los bailes modernos se realizaban "con

${ }^{63} \mathrm{Ibid} ., 74$. 
pretexto de favorecer las uniones legítimas" ya que en ellos se "multiplicaban las excitaciones sexuales". ${ }^{64}$

El último tema que tocaré en este apartado se refiere a la responsabilidad y función de los docentes, a quienes esta obra estaba dedicada. Recordemos que Chávez fue fundador en 1921 de la cátedra de psicología de la adolescencia impartida por él a maestros normalistas y alumnos de la Escuela Normal adscrita a la Escuela de Altos Estudios de la Universidad Nacional de México. Chávez ponía en sus manos la responsabilidad de formar a los adolescentes, futuros actores de la vida pública, para con ello contribuir a la regeneración social y a la restitución de la armonía que se había perdido en el mundo. ${ }^{65} \mathrm{El}$ maestro era además de formador, árbitro y vigilante. Debía impedir los abusos entre los jóvenes y conjuntamente con los padres de familia, tener la suficiente preparación para detectar, reportar y actuar frente a los problemas de conducta y desarrollo psicológico e intelectual de sus pupilos. ${ }^{66}$

\section{DETRÁS DEL TELÓN: BALANCE, CONSTRUCCIÓN Y RECEPCIÓN DE LA OBRA}

Como veremos en este apartado, Ensayo no tuvo en el ámbito académico internacional un impacto muy notable. A lo largo de sus páginas nuestro autor tampoco hace referencia a algún tipo de contribución realizada en el país en torno a la psicología experimental. ${ }^{67}$ Sin embargo, lo que resulta importante para nosotros, sus lectores contemporáneos, es que nos acerca a los contenidos de las cátedras

${ }^{64}$ Ibid., 175-176.

${ }^{65}$ Ibid., 13-14, 187.

${ }^{66}$ En Ensayo se propone el ajuste de los programas educativos a los alumnos con un desarrollo más lento o con características particulares, como el caso de las mujeres a quienes se les recomendaba no realizar cierto tipo de labores. Ibid., 124, 429, 419.

${ }^{67}$ Puedo nombrar la escueta referencia a las mediciones antropométricas sobre el desarrollo físico y mental de los niños mexicanos del médico Rafael Santamarina. Esta falta de reconocimiento en realidad no significó la inexistencia de esfuerzos por desarrollar esta rama de la psicología en México, podemos mencionar el trabajo de Enrique O. Aragón, quien fue alumno de Chávez en la Escuela Nacional Preparatoria y es considerado el fundador del primer laboratorio de psicología experimental en el país. López Ramos, Historia de una psicología, 123. 
que Chávez impartió entre 1921 y 1934 sobre psicología de la adolescencia en la Facultad de Altos Estudios de la Universidad Nacional de México.

Sería injusto restar mérito a la obra de Chávez, encasillándola únicamente como un trabajo recopilatorio de las ideas planteadas por otros investigadores, filósofos e intelectuales. Para algunos jóvenes, durante los treinta, el texto de Chávez fue el único medio del que dispusieron para el conocimiento de psicología y de sus avances a nivel internacional. Como bien lo expresó Mauricio Tenorio Trillo, la gran diferencia entre la evolución de las ciencias sociales en Estados Unidos y México fue el esfuerzo gubernamental del país angloparlante por impulsar entre sus jóvenes a especialistas que fueron enviados a estudiar a los países e instituciones extranjeras que protagonizaban los avances más significativos en sus respectivas áreas sociales. ${ }^{68}$ Por otro lado, en México, si bien estos esfuerzos también se llevaron a cabo, se realizaron de forma muy limitada. Los individuos que encabezaron la introducción de las ciencias sociales, ya con título profesional (generalmente como abogados, médicos o como profesores normalistas), fueron enviados y financiados por el gobierno mexicano como observadores de los sistemas de enseñanza y organización de instituciones en Europa y los Estados Unidos; o en otras ocasiones enviados a tomar algunos cursos en el extranjero. ${ }^{69}$ Parte de esta situación se derivó de la falta de recursos económicos del nuevo régimen gubernamental, quizá por ello la solución de algunas instituciones mexicanas fue la invitación a profesores extranjeros para ofrecer cátedras sobre sus especialidades en México, tratando de contribuir, de esta forma, a un mejoramiento

${ }^{68}$ Mauricio Tenorio Trillo, "Stereophonic Scientific Modernisms”, 1,156.

${ }^{69}$ Por ejemplo Claude Fell presenta datos más precisos: afirma que en 1922 la Universidad Nacional a través de la SEP otorgó 116 becas a mexicanos, enfatizadas para el estudio de las ciencias químicas. Claude Fell, José Vasconcelos, 299. Podemos encontrar por ejemplo que una comisión de maestros mexicanos fueron enviados a la Universidad de Columbia, "donde el Instituto de Educación Internacional les facilitaría la observación y práctica educativa de las escuelas secundarias americanas establecidas en Nueva York. El objetivo de esta visita era la implantación de una organización firme en materia de educación secundaria”. "Una comisión de maestros parte a los Estados Unidos”, $\mathrm{La}$ Revista, 7 de diciembre de 1925. 
académico y junto con ello, a la preparación de un número más amplio de jóvenes y a la organización de instituciones nacionales especializadas.

Como ya fue mencionado, Ensayo de psicología de la adolescencia apareció en 1928. Aunque impreso por la Editorial Cultura fue financiado por la Secretaría de Educación Pública. ${ }^{70}$ El tiraje fue de sólo 500 ejemplares, de los cuales cerca de sesenta fueron enviados a profesores e instituciones de investigación en el extranjero. Tanto la segunda edición (1956) como la tercera (1966) fueron publicadas después de la muerte de Chávez, ocurrida en 1946, por la editorial Jus considerada por muchos ańos como una institución conservadora, último reducto para la publicación de obras propias del conservadurismo mexicano. ${ }^{71}$ Además del traspaso de derechos de autor por su hija Leticia Chávez, que al igual que él era una católica fervorosa, el investigador Sergio López Ramos apunta que la formación profesional de psicólogos, su afiliación a las diferentes escuelas y la difusión de nuevas publicaciones sobre dicha disciplina en México promovió el destierro de la obra de nuestro autor. ${ }^{72}$

El trabajo hasta aquí expuesto deja claro que entre los logros más importantes del trabajo de Chávez, por lo menos para el estudio de

${ }^{70}$ Algunas editoriales mexicanas con una fuerte tradición habían cerrado sus puertas durante la contienda armada de la revolución, pero otras como Los Talleres Gráficos de la Nación, México Moderno, Editorial Cultura, Porrúa y hermanos y Andrés Botas e hijo llenaron la brecha abandonada por sus antecesoras.

${ }^{71}$ La editorial Jus surgió en 1933, de los intereses de algunos estudiantes de la Facultad de Derecho y Ciencias Sociales de la Universidad de publicar una revista de investigación alejada de las "politiquerías" en las que las autoridades universitarias estaban sumidas. A pesar del poco éxito de la primera época de esta publicación, Luis de Garay, uno de sus fundadores, insistiría a su profesor y exrector de la Universidad, Manuel Gómez Morin para su apoyo y orientación. Para el 15 de marzo de 1940, Jus se convertiría en una editorial, al publicar su primer libro intitulado La nacionalidad mexicana, de Eduardo Trigueros. Cabe destacar de Gómez Morín había sido alumno de Chávez en la ENP.

72 Sergio López Ramos, Historia de una psicología: Ezequiel Chávez Lavista (México: Plaza y Valdés editores, 1997), 127. La primera especialización en dicha disciplina en México fue la maestría en ciencias psicológicas impartida en la Escuela de Altos Estudios en 1939. La licenciatura en dicha institución fue establecida hasta 1960. Reidl Martínez Lucy María y María de Lourdes Echeveste García, comps., La Facultad de Psicología de la Universidad Nacional Autónoma de México: Treinta años a la vanguardia (México: UNAM, 2003), 22-30. 
caso que aquí nos compete, fue la introducción oficial de la psicología al ámbito académico mexicano. Por otro lado, contribuyó a centrar la atención de los sectores gubernamentales y del mundo educativo en México sobre la importancia y susceptibilidad del individuo durante la adolescencia. Acercó obras científicas, filosóficas, psicológicas, pedagógicas y literarias escritas en francés e inglés a un público monolingüe. Sus aportaciones no solo pueden contabilizarse a partir de las traducciones que realizó al idioma español, sino de la donación de volúmenes de libros cuyos pedidos solicitaba directamente de librerías en Francia y Estados Unidos, que eran por lo general novedades editoriales. ${ }^{73}$

En conclusión, el gran tributo de Chávez en Ensayo fue su intento por adaptar una multiplicidad de voces y propuestas de psicólogos, pedagogos y médicos internacionalmente reconocidos al contexto y necesidades mexicanas. Chávez aprovechó la coyuntura política y social que el conflicto revolucionario había abierto, para proponer la necesidad de una regeneración social y la construcción de adolescentes equilibrados, futuros ciudadanos de un México idílico. Fue entonces la defensa de la noción de internacionalismo que justificó la contribución internacional en la construcción del conocimiento (a través del intercambio académico y de investigaciones) en pos del mejoramiento de la sociedad mundial.

Las propuestas de Chávez no fueron las únicas difundidas en México durante principios del siglo. Otras más, formuladas por algunos profesores y especialistas como Andrés Osuna, Moisés Sáenz, Rafael Ramírez, José Ángel Ceniceros, Luis Garrido y Mathilde Rodríguez Cabo, entre otros, fueron escuchadas, discutidas y aplicadas

${ }^{73}$ Suyas son las primeras traducciones al español de Elements of Psychology de Edward B. Titchener (esp. 1904) y Resumen sintético de los principios de moral de Herbert Spencer (1910). Entre algunos de los documentos del Fondo Documental Ezequiel A. Chávez del Archivo Histórico de la UNAM, podemos encontrar recibos de los pedidos que Chávez realizó a librerías en Estados Unidos y Europa. Por ejemplo, pude encontrar una solicitud de compra para la Ginn and Company Publishers realizada por Chávez en 1921, donde solicitó 2 ejemplares de New Geography de Frye-Atwood; Principles of Argumentation de Baker y Huntington; Ethics for Young People de Everett; y 30 ejemplares de la Aritmética de Wentworth, entre otros. Ahunam, Ezequiel A. Chávez, caja 107, exp. 321, doc. 31-34, foja 65, año 1921. 
en políticas educativas y legislativas. ${ }^{74}$ Aunque el término "adolescente" fue inicialmente utilizado para referirse a los jóvenes de los sectores medios y bajos, y con ello los cuidados correspondientes para los individuos denominados como tales, a partir de los cuarenta la preocupación por este grupo de edad se extendió a los sectores populares y rurales. A pesar de ello, hoy es un reclamo generalizado el olvido del Estado por las necesidades y el futuro de los nińos y los adolescentes. Este abandono, según los reportes de algunas organizaciones no gubernamentales, además de la violencia intrafamiliar y las carencias materiales, han contribuido a la participación cada vez mayor de menores de edad en el crimen organizado que les ofrece reconocimiento y mejor calidad de vida. Faltan, en palabras de los menores y de los investigadores mismos, instituciones gubernamentales que legislen por la protección de este grupo de edad y que atiendan de manera profiláctica la violencia doméstica y escolar a la que se enfrentan cotidianamente nińos y adolescentes. ${ }^{75}$

\section{Fuentes:}

Archivo

Archivo Histórico de la unam (ahunam), fondo Ezequiel A. Chávez, México, unam (1921-1923)

\section{Hemerografía}

El Abogado Cristiano, México, 1913.

Boletín de Educación (SEP), México, noviembre 1915.

La Revista (Universidad Nacional), México, 1925-1926.

${ }^{74}$ Andrés Osuna y Moisés Saenz fueron en distintos momentos impulsores del establecimiento de la escuela secundaria en México que abriría los primeros planteles en 1926. Mathilde Rodríguez Cabo, José Ángel Ceniceros y Luis Garrido enfocaron sus esfuerzos en el estudio de la delincuencia infantil y juvenil y propusieron la importancia del tratamiento psicológico y reinserción de los jóvenes a la sociedad. Mathilde Rodríguez Cabo, Estudios sobre delincuencia e infancia abandonada (México: La razón, 1931); José Ángel Ceniceros y Luis Garrido, La delincuencia infantil en México (México: Ediciones Botas, 1936).

${ }^{75}$ Véase Elena Azaola, La reinserción socio-familiar de los adolescentes en conflicto con la ley: de la situación actual hacia una propuesta de intervención, http://www.crime-preventionintl.org/fileadmin/user_upload/membres/Reporte_Adolescentes_CIESAS_22sep14.pdf (Fecha de consulta: 11 de agosto de 2015). 
Bibliografía

Agostoni, Claudia. Monuments of Progress. Modernization and Public Health in Mexico City, 1876-1910. Calgary: University of Calgary Press, University Press of Colorado, UnAM, 2003.

Anaya Merchant, Luis. Ezequiel A. Chávez. Una aproximación biográfica a la historiografia de la rectificación. Aguascalientes: Gobierno del Estado de Aguascalientes, Instituto Cultural de Aguascalientes, 2002.

Ardila, Rubén. La psicología en América latina: pasado presente y futuro. México: Siglo XXI editores, 1998.

AzAOLA, Elena. La reinserción socio-familiar de los adolescentes en conflicto con la ley: de la situación actual hacia una propuesta de intervención. http://www.crime-prevention-intl.org/fileadmin/user_ upload/membres/Reporte_Adolescentes_CIESAS_22sep14.pdf (Fecha de consulta: 11 de agosto de 2015).

Barona, Josep Lluis, dir. Historia de la ciencia y de la técnica, núm. 46. Madrid: Akal, 1991.

Bazant, Milada. Historia de la educación durante el porfiriato. México: El Colegio de México, 2006.

Benjamin Jr., Ludy. A Brief Study of Modern Psychology. Malden: Blackwell Publishing, 2007.

Castillo Peraza, Carlos, recop. Manuel Gómez Morin: Constructor de instituciones. Antología. México: Fondo de Cultura Económica, 1994.

Ceniceros, José Ángel y Luis Garrido. La delincuencia infantil en México. México: Ediciones Botas, 1936.

Corominas, Joan de. Diccionario crítico etimológico de la lengua castellana. Madrid: Gredos, 1976.

Chávez, Ezequiel A. Ensayo de psicología de la adolescencia. México: Jus, 1956.

Chávez, Leticia. Recordando a mi padre. Ezequiel A. Chávez. México: Asociación Civil "Ezequiel A. Chávez, 1967.

FeLl, Claude. José Vasconcelos: los años del águila, 1920-1925. México: UNAM, 2009.

Garcíadiego Dantán, Javier. Rudos contra cientificos. La Universi- 
dad Nacional durante la Revolución Mexicana. México: El Colegio de México, 1996.

"De Justo Sierra a Vasconcelos. La Universidad Nacional durante la Revolución Mexicana”. Historia Mexicana. Homenaje a don Edmundo O'Gorman 46(4) (abril-junio 1997): 769-819.

Gilfoyle, Timothy. "Street-rats and Gutter-snipes: Child Pickpockets and Street Culture in New York City, 1850-1900. Journal of Social History 37(4) (verano 2004): 853-882.

Gómez IzQuIERDo, José Jorge, coord. Los caminos del racismo en México. México: Plaza y Valdés, 2005.

Gumbrecht, Hans Ulrich. Producción de presencia. México: Universidad Iberoamericana, 2005.

Hale, Charles. La transformación del liberalismo en México a fines del siglo XIX. México: Fondo de Cultura Económica, 2002.

Krauze, Enrique. Caudillos culturales en la Revolución Mexicana. México: Siglo XXI editores, 2000.

Knight, Alan. "Popular Culture and the Revolutionary State in México, 1910-1940". Hispanic American Historical Review 74(3) (agosto 1994): 393-444.

Levi, Giovanni y Jean Claude Schmitt. Historia de los jóvenes, 2 vols. Madrid: Taurus, 1996.

López Austin, Alfredo. Educación mexica. Antología de textos sahaguntinos. México: IIA-UNAM, 1985.

López Ramos, Sergio. Historia de una psicología: Ezequiel Chávez Lavista. México: Plaza y Valdés editores, 1997.

Loyo, Engracia. Gobiernos revolucionarios y educación popular en México, 1911-1928. México: El Colegio de México, 1999.

Mauriac, François. Le Jeune Homme. París: éd. Hachette, 1926.

Mendoza Rojas, Javier. Los conflictos de la UNAM en el siglo XX. MéxiCO: UNAM-CESU, 2001.

Meyer, Alicia, coord. México en tres momentos: 1810-1910-2010. Vol. 2. México: IIH-unam, 2007.

Meza Huacuja, Ivonne. La edad dificil. Los adolescentes modernos en la ciudad de México: 1876-1934. Tesis de Doctorado, El Colegio de México, 2015.

Meza Medina, Gustavo. La creación de la SEP: entre Chávezy Vas- 
concelos. http://www.comie.org.mx/congreso/memoriaelectronica/v09/ponencias/at09/PRE1 178325894.pdf (Fecha de consulta: 1 de junio de 2012).

Puente Lutteroth, María Alicia. Movimiento cristero: una pluralidad desconocida. México: Editorial Progreso, 2002.

Reidl Martínez, Lucy María y María de Lourdes Echeveste García. La Facultad de Psicología de la Universidad Nacional Autónoma de México: treinta años a la vanguardia. México: unam, 2003. Rizquez, Fernando. Psiquiatría y homeopatía. Nueva Dehli: B.Jain Publishers, 2005.

RocKWELl, Elsie. Hacer escuela, hacer estado. La educación posrevolucionaria vista desde Tlaxcala. México: El Colegio de Michoacán, Ciesas, Cinvestav, 2007.

Saladino García, Alberto, comp. Humanismo mexicano del siglo XX. Vol. I. Toluca: Universidad Autónoma del Estado de México, 2004.

Savage, Jon. Teenage. The Creation of Youth Culture. Londres: Viking Penguin, 2007.

Secretaría de Educación Pública (sep). El esfuerzo educativo en México. México: SEP [¿1929?].

Silva Herzog, Jesús. El agrarismo mexicano y la reforma agraria. México: Fondo de Cultura Económica, 1974.

Tenorio Trillo, Mauricio. "Stereophonic Scientific Modernisms: Social Science between Mexico and the United States, 1880s1930s". The Journal of American History. The Nation Beyond: Transnational Perspectives on United States History: Special Issues 86(3) (diciembre 1999): 1,156-1,187.

TORRE, Renée de la et al., Los rostros del conservadurismo mexicano. México: Editorial La Casa Chata, 2005.

Urías Horcasitas, Beatriz. Historias secretas del racismo en México (1920-1950). México: Tusquets, 2007.

VArios autores. Historia general de México. México: El Colegio de México, 2010.

Velázquez Andrade, Manuel. La delincuencia juvenil. México: Editorial Cultura, 1932. 